\title{
Cervical Ganglioneuroma in Pediatric Age: A Case Report
}

\author{
Case Report Antonio F Lima, Filipa C Moreira, Ana Menezes, Luís Dias \\ Department of Otorhinolarynogology, Braga Hospital, Braga, Portugal
}

Abstract

Cite this article as: Lima AF, Moreira FC, Menezes A, Dias L. Cervical Ganglioneuroma in Pediatric Age: A Case Report. Turk Arch Otorhinolaryngol 2018; 56(4): 237-40.

This study was presented at the ENT World Congress, June 24-28, 2017, Paris, France.

\section{Corresponding Author:}

Antonio F Lima; antoniofonteslima24@gmail.com

Received Date: 26.07.2018

Accepted Date: 12.10 .2018

(c) Copyright 2018 by Official Journal of the Turkish Society of Otorhinolaryngology and Head and Neck Surgery Available online at www.turkarchotolaryngol.net DOI: $10.5152 /$ ta0.2018.3690
Ganglioneuroma is a rare, benign, non-invasive tumor emerging from the sympathetic system. Of these tumors, only $8 \%$ occur in the neck. In this report, we present a case of a 13-year-old girl with a 2-year history of enlarging neck mass. Her only complaint, aside from neck swelling, was dysphagia. Physical and radiological examinations revealed a large mass centered in the right carotid space. A transcervical approach was used to excise the tumor emerging from the sympathetic ganglia. The patient developed tem- porary Horner's syndrome postoperatively. In a few weeks, she was completely asymptomatic. Histological examination was compatible with ganglioneuroma. Surgical excision is the only definite treatment of cervical ganglioneuroma and is also the only way to confirm the diagnosis. Injury during surgery may result in significant morbidity.

Keywords: Ganglioneuroma, neuroblastoma, neurogenic neck tumors, benign cervical masses, pediatric neck tumors

\section{Introduction}

Cervical masses in pediatric patients are usually classified into one of the three categories: developmental, inflammatory/reactive, or neoplastic (1). Onset and duration of symptoms, exposures (animals and other sick children), and location can aid in the differential diagnosis. Neoplastic lesions can be either benign or malignant, with the latter being rare in this age group (2).

Ganglioneuroma, together with ganglioneuroblastomas (intermixed and nodular) and neuroblastomas, comprises a group of tumors called peripheral neuroblastic tumors (3-5). It is a rare, benign, non-invasive tumor which usually emerges from the sympathetic ganglia. It is most frequently located in the abdomen (posterior mediastinum, retroperitoneum, and adrenal glands) in $65 \%-80 \%$ of cases, followed by the thorax in $10 \%-15 \%$, with the neck region as its rarest location in $5 \%-8 \%$ of patients $(6,7)$. It typically presents as an enlarging mass of the neck. Its symptoms usually stem from mass effect and nerve dysfunction.

\section{Case Presentation}

A 13-year-old girl presented for evaluation at the otorhinolaryngology department with a history of a 2-year enlarging neck mass. She had previously been followed up by a pediatric surgeon and had undergone echo-guided biopsy, which was inconclusive. She had recently started with complaints of dysphagia. On examination, a medial bulging of the right lateral pharyngeal wall accompanied by cervical right swelling was noted. It had normal overlying skin and was elastic in consistency, tender, and mobile. Magnetic resonance imaging (MRI) showed a mass of $35 \times 60 \mathrm{~mm}$ centered in the right carotid space (Figure 1,2). The mass displaced anteriorly the internal and external carotid arteries and collapsed posteriorly the internal jugular vein. Blood and urine results were negative for by-products of sympathetic response, including vanillylmandelic acid and hydroxymandelic acid. Surgical excision was planned.

Under general anesthesia, a transcervical approach was used to excise the tumor (Figure 3 ). The vagus nerve was identified and preserved, as were the ac- 


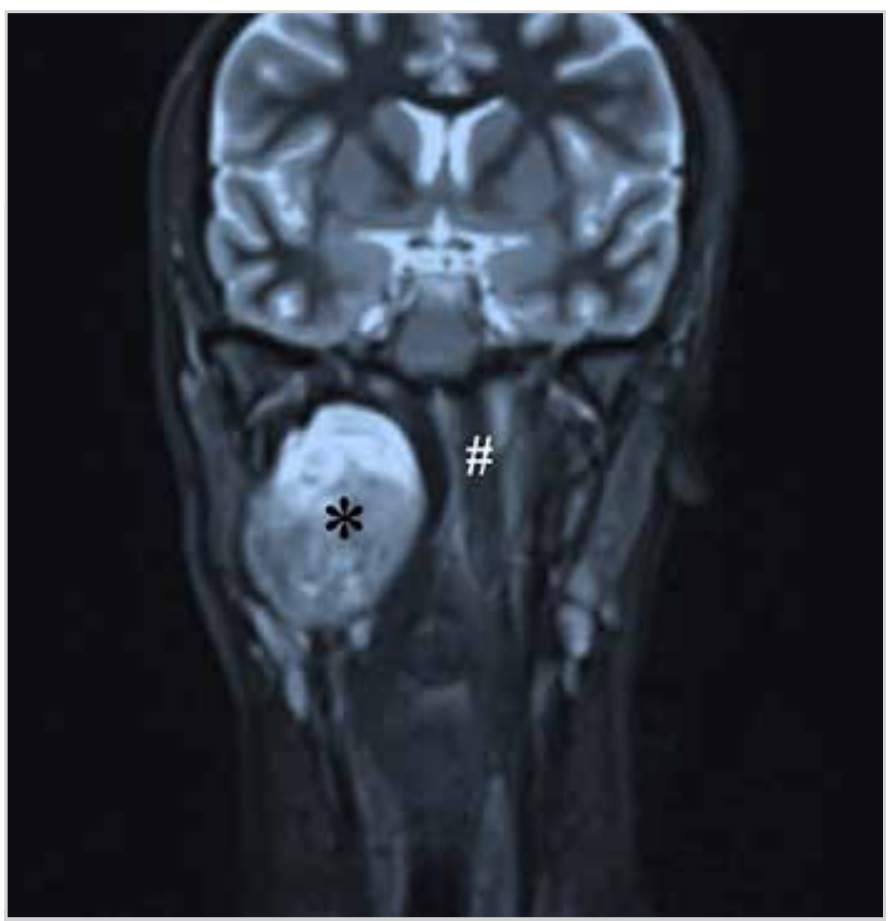

Figure 1. MRI (T2) coronal section shows a mass of $35 \times 60 \mathrm{~mm}$ centered in the right carotid space, extending to the skull base *tumor, \#: pharynx

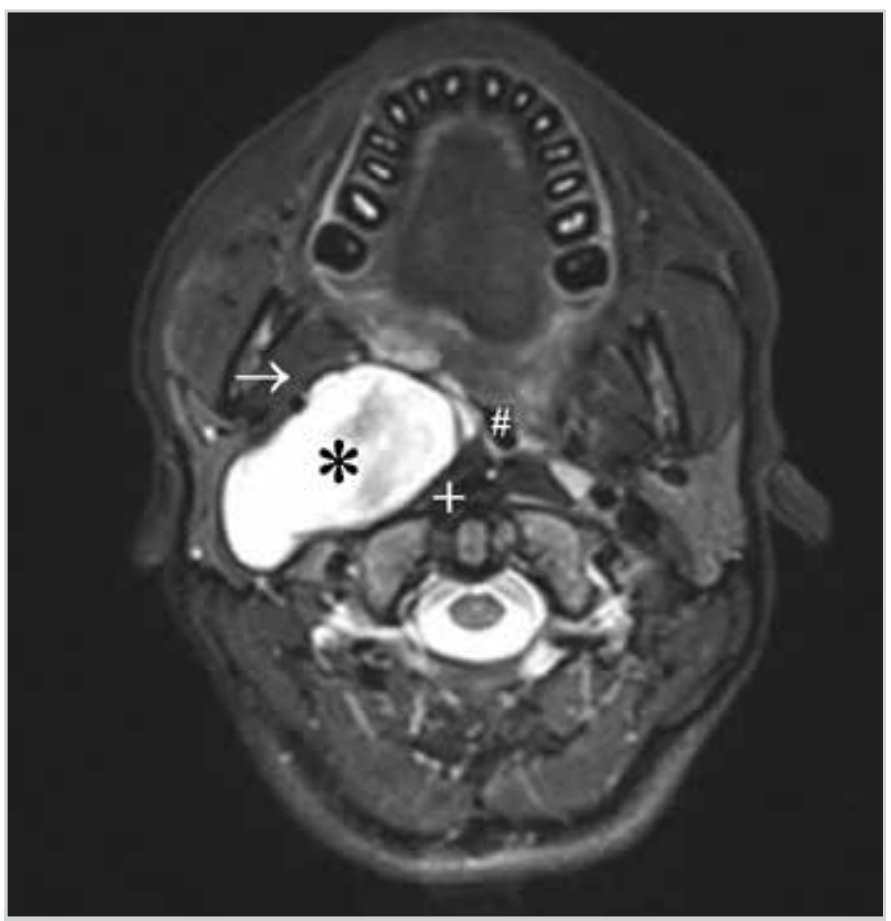

Figure 2. MRI (T2) axial section shows a mass of $35 \times 60 \mathrm{~mm}$ centered in the right carotid space, extending to the skull base *tumor, +: internal jugular vein, $\rightarrow$ : carotid artery, \#: pharynx

cessory and hypoglossal nerves. The internal jugular vein and the common carotid artery which were displaced by the tumor were retracted and also preserved. The tumor was derived from the sympathetic chain, which ramified into the mass. The excised lesion was well circumscribed, making an intact removal possible (Figure 4). In spite of being in continuity with the sympathetic chain, this was left intact.

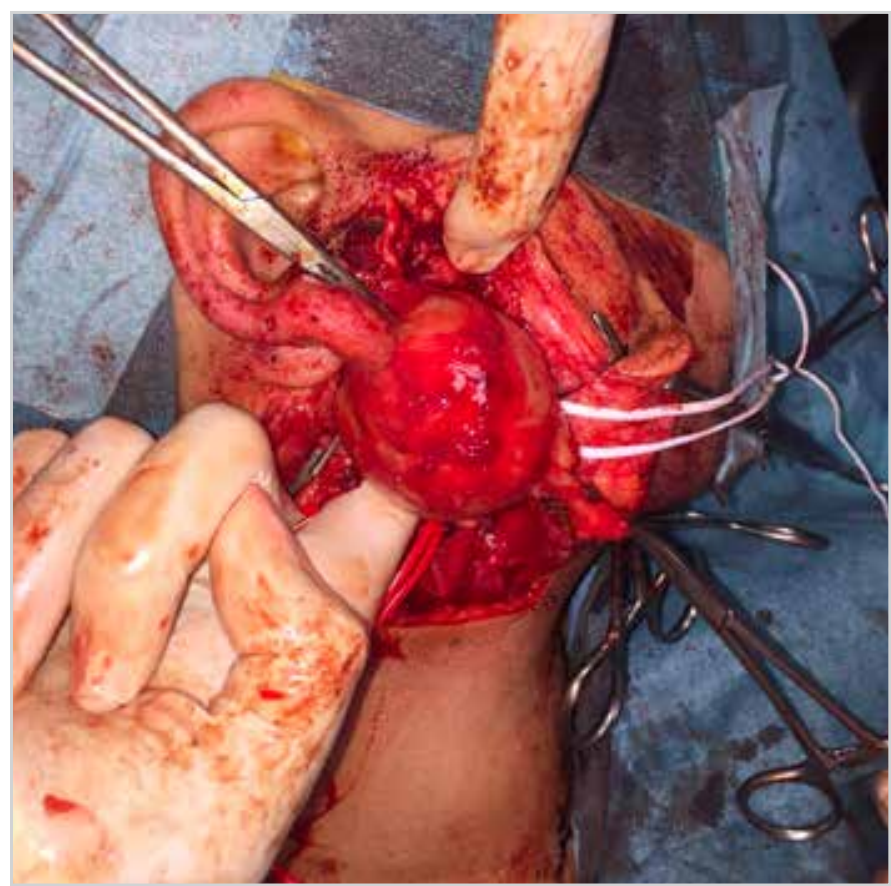

Figure 3. A well-circumscribed mass which emerged from the sympathetic chain

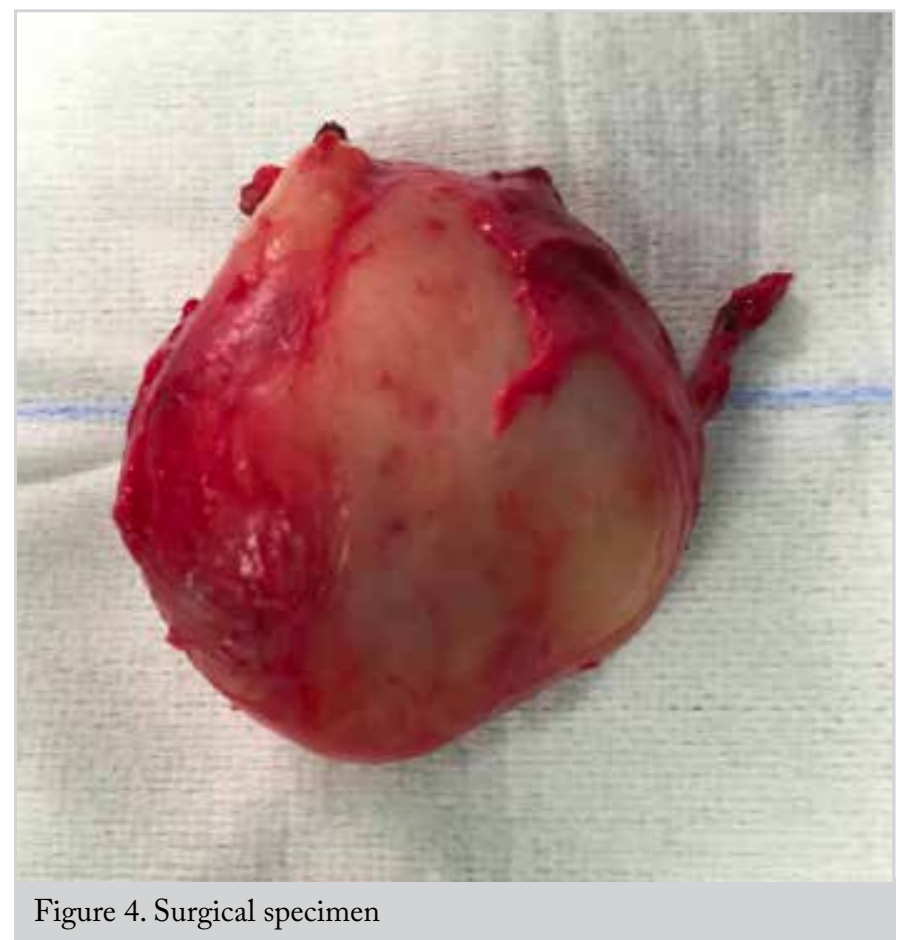

The patient was extubated immediately after the surgery with no clinical signs of respiratory distress. The patient developed temporary Horner's syndrome postoperatively, characterized by ipsilateral ptosis, myosis, and asymmetry of the lower eyelid, with full recovery within the first month postoperatively. Histopathological examination revealed ganglioneuroma(Figure 5). The patient has been followed up for 24 months with no signs of recurrence. Written informed consent was obtained for this case report from the parents of the patient. 


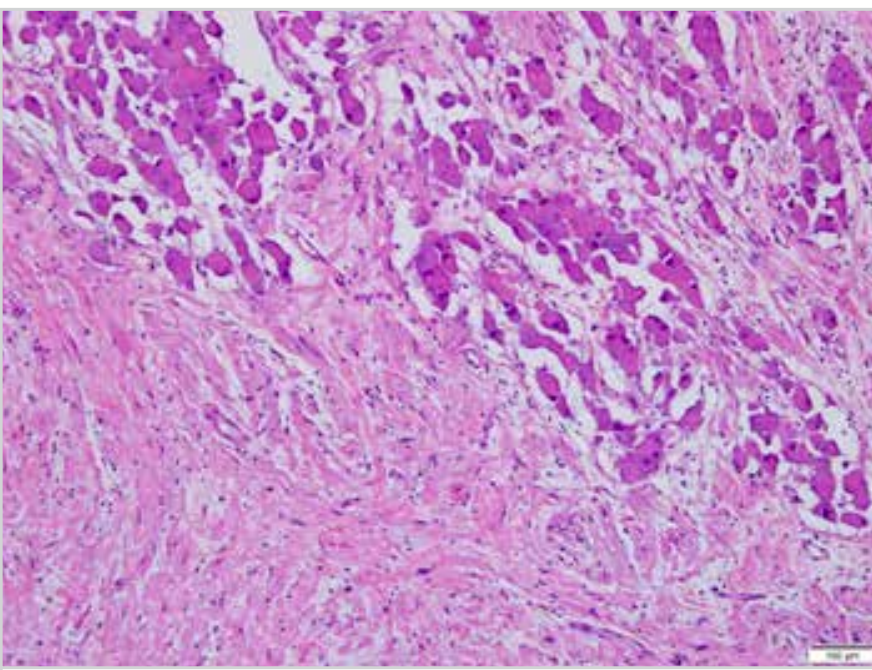

Figure 5. Microscopic examination revealed ganglioneuroma, namely clusters of mature ganglion cells and intersecting bundles of spindle cells (H\&E, X100)

\section{Discussion}

Ganglioneuroma was first described by Loretz in 1870 (8). It originates from the primordial neural crest cells which are undifferentiated cells of the central nervous system $(6,7)$. These cells are present in the sympathetic ganglia and adrenal medulla, indicating that these tumors may grow nearly anywhere along their distribution.

Ganglioneuroma usually occurs in the abdomen or thorax, but it can also occur in the head and neck region, with the latter being its least common location (6). Actually, this is one of the rarest neck tumors (6-10). The first report of a ganglioneuroma located in the neck was made in 1899 by de Quervain (8). The most frequent origin in the neck region is the cervical sympathetic chain, but it can also be located in the larynx, pharynx, and ganglion nodosum of the vagus nerve (9).

These types of tumors are more common at a young age, as 60\% of patients with ganglioneuroma are $<20$ years old $(6,10)$. The average age of presentation is 11 years old, with a slight preponderance in the female gender (10).

They are usually slow-growing masses, with most of them being silent in the early stages of development (6). Their symptoms are a consequence of compression of the neighboring structures, as in the descripted case who presented with dysphagia. Globus sensation, dyspnea, voice change, and nasal congestion can be due to the tumors' expansion. They can also cause nerve dysfunction.

The reviewed literature suggests the possibility of these tumors being secretory; however, our case and the ones reviewed were not metabolically active (7-10). Nevertheless, the differential diagnosis between ganglioneuroma and neuroendocrine tumors can be quite difficult. According to the North American Neuroendocrine Tumor Society guidelines, the initial testing for pheochromocytoma or paraganglioma must include measure- ments of fractionated metanephrines in plasma, urine, or both, as available. Given their high sensitivity for neuroendocrine tumors (reaching 100\% in children and 99\% in adults), they are part of the initial routine evaluation of this kind of masses (11).

In other case series, fine-needle aspiration failed to identify this kind of tumor in $60 \%$ of cases (10). Nevertheless, it can be of tremendous value in the differential diagnosis in pediatric patients, as it is a rapid, safe and cost-effective method, although sedation is often required (7).

In addition, imaging is crucial not only on the differential diagnosis of the neck mass but also to plan surgery. Generally, both computed tomography (CT) and MRI are mandatory. CT frequently reveals a well-defined mass, which displaces the surrounding structures (6). MRI shows intermediate signal intensity and cystic or non-homogeneous contrast enhancement. On the other hand, functional imaging with metaiodobenzylguanidine scintigraphy has a high specificity for neuroendocrine tumors and can aid in the precise localization of that kind of disease. Several authors report having used it preoperatively for differential diagnostic purposes. This examination was not used in our patient as the by-products of sympathetic response were negative (11). As in our case, preoperative diagnosis is not possible most of the times.

Surgical resection of these tumors is the gold standard treatment (6-10). The approach may vary. As in our case, a transcervical approach can be used; however, transoral, transparotid, transcervical-transpharyngeal, and infratemporal fossa approach can also be used depending on the location, size, and pathological type (10). Mandibulotomy has also been performed in a few cases for better exposure of the skull base (10). Damage to the nearby neural and vascular structures during surgery may result in significant morbidity (8). However, these symptoms tend to resolve rapidly (7).

Definitive diagnosis is based on histopathological analysis. The International Neuroblastoma Pathology Classification defines four categories of peripheral neuroblastic tumors characterized by the grade of neuroblastic differentiation and the degree of Schwannian stromal development (5). Neuroblastomas are cellular neuroblastic tumors without prominent Schwannian stroma. Ganglioneuroblastomas are subdivided into intermixed and nodular categories. The former comprises neuroblastic elements in an abundant Schwannian stroma, and the latter is a composite tumor with a neuroblastic nodular component and either an intermixed ganglioneuroblastoma or a ganglioneuroma component. As in our case, ganglioneuromas represent the most differentiated form, consisting of mature ganglion cells distributed in a predominant Schwannian stroma. Ganglion cells vary in distribution and number and can be either localized or widely scattered. Schwann cells may ensheath neuritic processes or may be arranged in small intersecting fascicles, which are separated by loose myxoid stroma. Neuroblasts should be negative in order to make this diagnosis. Furthermore, significant atypia, mitoses, or necrosis must be absent $(4,5)$. 
If the diagnosis is confirmed after surgical excision, it can be curative. The prognosis is favorable, as ganglioneuromas do not have metastatic potential (10). The patient does not require further treatment. Even in the case of incomplete resection, it does not have the potential to recur (5). Radiation should be avoided in these cases, especially in young ages, as it can cause growth retardation and later problems (6). On the other hand, other categories of neurogenic tumor have different behaviors. Neuroblastoma is a malignant solid tumor, and ganglioneuroblastoma has moderate malignant potential, which may require the need for close follow-up and for complimentary treatment, such as chemo- or radiotherapy.

Moreover, isolated ganglioneuromas can also occur in association with at least two syndromes, namely multiple endocrine neoplasia type $2 \mathrm{~B}$ and type I neurofibromatosis (10). A family history of any of these two should be assessed, and if positive, further study is recommended.

\section{Conclusion}

Surgical excision is the only definite treatment of cervical ganglioneuroma to prevent further growth and compression of the neighboring structures. It is also the only way to confirm the diagnosis. These tumors are usually not aggressive. Injury during surgery may result in significant morbidity.

Informed Consent: Written informed consent was obtained from the parents of the patient.

Peer-review: Externally peer-reviewed.

Author Contributions: Concept - A.F.L., F.C.M., A.M., L.D.; Design - A.F.L., F.C.M., A.M., L.D.; Supervision - A.F.L., F.C.M., A.M., L.D.; Resource - A.F.L., L.D.; Materials - A.F.L., F.C.M., A.M., L.D.; Data Collection and/or Processing - A.F.L., L.D.; Analysis and/or Interpretation - A.F.L., F.C.M., A.M., L.D.; Literature Search - A.F,L., A.F.L.; Writing - A.F.L., F.C.M.; Critical Reviews - A.F.L., F.C.M., A.M., L.D.

Acknowledgements: The authors acknowledge the help from the departments of Neuroradiology and Pathology of Hospital de Braga for providing the images of the exams.
Conflict of Interest: The authors have no conflicts of interest to declare.

Financial Disclosure: The authors declared that this study has received no financial support

\section{References}

1. Meier JD, Grimmer JF. Evaluation and management of neck masses in children. Am Fam Physician 2014; 89: 353-8.

2. Torsiglieri AJ, Tom LW, Ross AJ III, Wetmore RF, Handler SD, Potsic WP. Pediatric neck masses: guidelines for evaluation. Int J Pediatr Otorhinolaryngol 1988; 16: 199-210. [CrossRef]

3. Lonergan GJ, Schwab CM, Suarez ES, Carlson CL. Neuroblastoma, ganglioneuroblastoma, and ganglioneuroma: radiologic-pathologic correlation. Radiographics 2002; 22: 911-34. [CrossRef]

4. Antonescu C, Scheithauer B, Woodruff J. AFIP Atlas of Tumor Pathology. Tumors of the peripheral nervous system. Silver Spring, MD. 4th series. Fascicle 19. American Registry of Pathology; 2013.p.452-4.

5. Lloyd R, Osamura R, Klöppel G, Rosai J, Bosman F, Jaffe E et al. WHO classification of tumours of endocrine organs. 4th Edition, Volume 10.

6. Califano L, Zupi A, Mangone FM, Long F. Cervical ganglioneuroma: Report of a case. Otolaryngol Head Neck Surg 2001; 124 : 115-6. [CrossRef]

7. Dutta H. Cervical ganglioneuroma in a child. SM J Pediatr Surg 2016; 2: 1013.

8. Kaufman MR, Rhee JS, Fliegelman LJ, Costantino PD. Ganglioneuroma of the parapharyngeal space in a pediatric patient, Otolaryngol Head Neck Surg 2001; 124: 702-4. [CrossRef]

9. Gopal A, Singh S, Sridhar M, Khurana N. Cervical Sympathetic chain ganglioneuroma: case report and review of literature. IOSR J Pharm 2015; 5: 8-11.

10. Cannady SB, Chung BJ, Hirose K, Garabedian N, Van Den Abbeele T, Kontai PJ. Surgical management of cervical ganglioneuromas in children. Int J Pediatr Otorhinol 2006; 70: 287-94. [CrossRef]

11. Chen H, Sippel RS, O'Dorisio MS, Vinik AI, Lloyd RV, Pacak K, et al. The North American Neuroendocrine Tumor Society consensus guideline for the diagnosis and management of neuroendocrine tumors: pheochromocytoma, paraganglioma, and medullary thyroid cancer. Pancreas 2010; 39: 775-83. [CrossRef] 